\title{
Editorial
}

\section{Being Digital, Digital Being}

\author{
Michael A PETERS | ORCID: 0000-0002-1482-2975 \\ Distinguished Professor, Faculty of Education, Beijing Normal University, \\ China PR \\ mpeters@bnu.edu.cn \\ ZHUXudong \\ Professor and Dean, Faculty of Education, Beijing Normal University, \\ China PR \\ zhuxd@bnu.edu.cn
}

We are pleased to see the publication of this special issue of The Beijing International Review of Education on the theme of 'Digital Youth: Living, Learning and Literacy' edited by Dr. LIN Ke and Professor Boris ZIZEK. The issue focusses on a significant topic for education and for youth and it spans ontological issues of everyday digital interaction, virtual friendship and loneliness, and digital self-portrayal of youth, as well as questions of digital literacy, digital curation among learners and engagement, together in consideration of the theme of global citizenship in a digital frame. These are big questions and represent the cutting edge of the phenomena of everyday digital existence for youth, an inescapable horizon for youth growing up digitally in a digital world that impact on all aspects of being human in the early twenty-first century. These are weighty topics that embrace being digital and digital being for youth in China and around the world. Research work has been ongoing around these and similar topics since the Internet went global in the early 1990 so so it is good to have an issue that examines 'Living, Learning and Literacy'.

Nicholas Negroponte (1995) almost twenty-five years ago outlined the history of digital technologies in his Being Digital predicting that we will move 
toward an entirely digital society where youth demand instantaneous results and structures the world through the four forces of the digital age: decentraliziation, globalization, harmonization, and empowerment. In a word the new realm of human experience digital that as an ensemble of digital technologies now creates and recreates us, especially digital youth who seem more adept and more creative than others, in terms of 'bits' represented by numbers is and os binary numbers that in their infinite sequence and arrangement shaped all forms of communication, entertainment, socialisation and education. The advent of the mobile phone with more power than original mainframes has permanently changed human society and cultural evolution since the first models were introduced in the 1980 os and now define our cybernetic subjectivities and personalities in cellular networks.

Who would have thought that the digital would be our saviour in the COVID-19 pandemic. It revealed both the lack of readiness in preparation of digital systems as fail-safe national systems in communication, production of knowledge, and education. While digital technologies have been adopted societally in almost every facet of existence few countries or institutions were ready for the effects of COVID-19. Digital technologies surely provided the means to survive the pandemic and to carry on, albeit in digital mode, but with varying degrees of success. We suspect that 'digital youth', that group (the first) that has grown up with digital technologies in the last twenty-five years, fared better than the older generation - this is our working hypothesis. Research on the flows of internet traffic might substantiate this hunch and might explain how quickly digital youth responded and utilized the internet and mobile technologies to communication with their friends and family.

'Digital youth' and 'being digital' refer to the changing ontological character that define the spaces within which identities are formed and developed but 'being digital' can refer to new forms of governmentality and cyber subjects with greater variation in formation of 'the social' - social media groups that form on the basis of age, gender, race, class, and interest and run across all categories abstracting from the social context often to add a layer to new visual identities supported by the visual based on videos and images. Such digital governmentalities also include the world of politics and policy attempts to capture, engage, and involve young people informs new digital forms of national solidarity. It also includes netizens or 'net citizens' that grow up and participate online. The terms wăngmín and wăngyǒu are used in China to refer Internet users and friends online. The early assessments by Negroponte and others may have been too optimistic is celebrating 'participatory cultures' of an emerging technological cultures and perhaps also overestimating the educational benefits while minimising its disadvantages. 
Digitalism - the condition of being born into and living in a digital culture based on continuous contact and learning to communicate online rather than in face-to-face relationships needs close examination. Within the theoretical framework of information theory that takes the forms of cosmological principles in information physics and throws information science into the complex interplay with the new biology - the two primary forces shaping the cultural world today - the future of humanity takes a decisive digital turn. As part of this 'bioinformationalism' education is also transformed when using AI technologies smart or 'intelligent' devices are produced also as digital beings with the prospects of organising, controlling, and educating youth.

We think that Bernard Stiegler (2013), a philosopher and head of the Institut de recherche et d'innovation (IRI), which he founded in 2006 at the Centre Georges-Pompidoun, provides an encompassing and persuasive overview when he writes: "The digital constitutes a new épistémè: it is the very nature of knowledge in all its forms that will be affected. This technology will function for our epoque in the same way that writing did for antiquity.'

It surely is a new épistémè involving new ways of knowing, of writing and reading, of forms of open education and pedagogy and of research. At the same time, it also is a new form of ontology involving new ways of being which is both relational and social and surfaces in related ideas of 'social capital,' 'situated learning,' and ' $\mathrm{P} 2 \mathrm{P}$ ' (peer-to-peer) accounts of commons-based peer production as a product of social and networked environments - rich semiotic and intelligent environments in which everything speaks.

We are appreciative of Ke Lin and Boris Zizek's efforts in bringing together this international collection of papers, and the contributors, that address aspects of the one of the most important themes facing youth, education and pedagogy, especially in the times of COVID-19 that has highlighted the digital turn and made it even more urgent in the building of new forms of sociality. We hope that you enjoy reading these papers as much as we did.

1 'The Digital Future of the University' at http://www.samkinsley.com/2014/01/15/the-digital -future-of-the-university-stiegler/. 